\title{
Carbon-enhanced stars in SDSS DR-3
}

\author{
T. Sivarani, B. Marsteller, \& T.C. Beers ${ }^{1}$ \\ ${ }^{1}$ Department of Physics and Astronomy \& Joint Institute for Nuclear Astophysics (JINA), \\ East Lansing, Michigan State University 48824-2320, USA \\ email: thirupathi@pa.msu.edu, marsteller@pa.msu.edu, beers@pa.msu.edu
}

\begin{abstract}
We present new carbon-enhanced stars identified from the third public release of the Sloan Digital Sky Survey, SDSS-DR3. We have generated synthetic spectra with varying carbon abundances, and compare them with the SDSS spectra. We have also performed a preliminary analysis of s-process enhancement in several SDSS carbon-enhanced stars. Spectral features that are sensitve to stellar luminosity and temperature have also been explored. These methods will be applied to the large set of public SDSS data, as well as to the forthcoming data from SEGUE, the Sloan Extension for Galactic Understanding and Evolution, in order to study carbon enhancement at different metallicities, the fraction of s-process enhancement that occurs in carbon-enhanced stars, and possibly isotopic carbon abundances and nitrogen abundances.
\end{abstract}

Keywords. Stars: carbon, stars: abundance, stars: abundance

\section{Introduction}

Recent surveys of very metal-poor stars, such as the HK survey of Beers and colleagues (Beers, Preston, \& Shectman 1992) and the Hamburg/ESO survey of Christlieb and collaborators (Christlieb 2003), have indicated a high frequency of carbon-enhanced stars at low metallicities. High-resolution follow-up studies have shown a variety of abundance patterns among these stars. In addition, a large number of faint high-latitude carbon stars have already been discovered from the Sloan Digital Sky Survey (SDSS; e.g., Margon et al.; Downes et al. 2004).

\section{Analysis and results}

In order to analyse large data sets such as SDSS, one needs to explore the use of automated techniques. For this purpose, we have generated theoretical spectra using ATLAS9 models (Castelli, Gratton, \& Kurucz 1997) with no overshoot approximation, and using the turbospectrum spectrum synthesis code (Plez, private communication). Theoretical spectra were calculated for temperatures from $3500 \mathrm{~K}$ to $6000 \mathrm{~K}$ and $\log \mathrm{g}$ from 0.0 to 5.0 for various $[\mathrm{C} / \mathrm{Fe}]$ and $[\mathrm{Fe} / \mathrm{H}]$ values. These grids are used to estimate the stellar parameters, carbon abundances, and to derive, or put limits on, s-process abundances in these stars. We have also used these synthetic grids to explore line indices that are luminosity and temperature sensitive.

\subsection{Luminosity Indicators}

The relative line depths of the $\mathrm{C}_{2} 5167 \AA$ band head and the Mg I triplet vary with log g. The $\mathrm{C}_{2}$ band is stronger for higher luminosities, while the $\mathrm{MgI}$ is weaker at higher luminosities. However, these indices depend on the $[\mathrm{C} / \mathrm{Fe}]$ values and temperature, which could be constrained from the MgI triplet line itself and also from the CrI $5206 \AA$ feature, which is also sensitive to luminosity. At solar metallicities and high carbon abundance $(\sim[\mathrm{C} / \mathrm{Fe}]=+1.0)$, the $\mathrm{C}_{2}$ features are saturated, and the line depths are no longer 

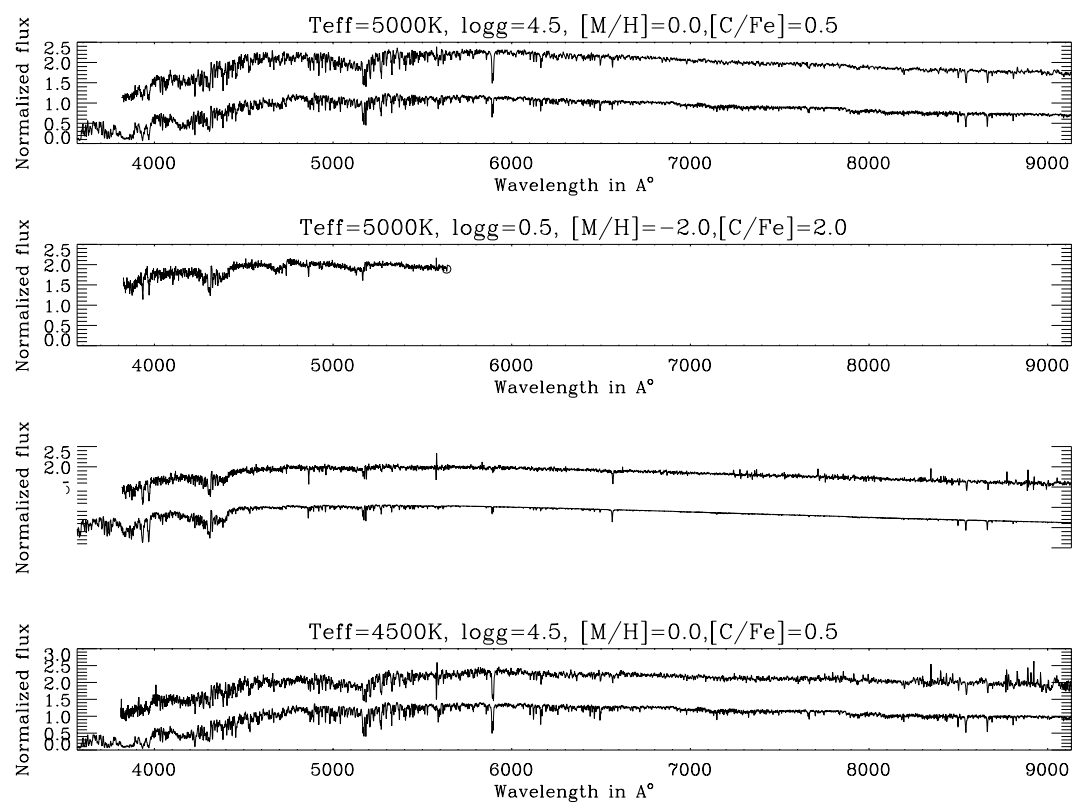

Figure 1. Comparision of observed SDSS spectra and the best matching synthetic spectra.

Each panel shows the SDSS spectra on the top and the synthetic spectra at the bottom.

sensitive to luminosity. The NaI D lines are quite sensitive to luminosity as well as to temperature. Jorgensen, Carlsson, \& Johnson (1992) show that the IR CaII triplet line strength increases with decreases in $\log g$ at solar metallicity. We have found that a comparision of CaI $5270 \AA$ and the CaII triplet is sensitive to luminosity.

Figure 1 shows a comparison of observed SDSS spectra and synthetic spectra which provided the best match.

\section{Conclusions}

Based on tests conducted to date, we expect that SEGUE will identify several thousand carbon-enhanced stars; several hundred will be sufficiently bright for high-resolution folow-up spectroscopic study. This dramatic increase in the number of carbon-enhanced metal-poor stars will enable a better understanding of early star formation and chemical evolution.

\section{Acknowledgements}

The authors are grateful for partial support from PHY 02-16783, Physics Frontier Centers/JINA: Joint Institute for Nuclear Astrophysics, awarded by the US National Science Foundation.

\section{References}

Beers, T.C., Preston, G.W., \& Shectman, S.A. 1992, AJ 103, 1987

Castelli, F. Gratton, R. \& Kurucz, R. 1997, A\& A 328, 841

Christlieb, N. 2003, Reviews in Modern Astronomy 16, 191

Downes, R.A., et al. 2004, AJ 127, 2838

Jorgensen, U.G., Carlsson, M., \& Johnson, H.R. 1992, A\&SA 254, 258

Margon, B., et al. 2002, AJ 124, 1651 